\title{
The overshadowing of instrumental conditioning by a stimulus that predicts reinforcement better than the response
}

\author{
ROBERT ST. CLAIRE-SMITH \\ Wilfrid Laurier University, Waterloo, Ontario N2L 3C5, Canada
}

\begin{abstract}
In an experiment designed to demonstrate the overshadowing of appetitive instrumental conditioning, three groups of rats were given 10 sessions of RI (random interval) training in which reinforcement was delivered $450 \mathrm{msec}$ following the response. The correlated group experienced a stimulus during the response-reinforcer delay interval, while an uncorrelated control group experienced a similar brief stimulus occasionally following responses, but these were not responses that typically produced reinforcement. A no-stimulus control group did not experience the brief response-produced stimulus. The experiment was run in two replications. The first employed a light as the critical stimulus, the second a tone. Over the $10 \mathrm{RI}$ sessions, subjects in the two control conditions increased their rate of responding significantly faster than subjects in the correlated condition in both replications. This finding was interpreted as an instance of the overshadowing of the acquisition of signal value by a response because of the presence of the stimulus, which, in the correlated condition, was a more reliable predictor of reinforcement than the response. A subsequent conditioned reinforcement test confirmed that, in the correlated condition, the stimulus had, indeed, become a signal for reinforcement as a function of RI training.
\end{abstract}

The effect of arranging a contingency between a response and a reinforcer can be conceptualized as depending on the response becoming a signal for the reinforcer, in the same sense that the effect of arranging a contingency between a stimulus and a reinforcer has been described as depending on the stimulus becoming a signal for the reinforcer (e.g., Mackintosh, 1975). This view encourages speculation that classical conditioning and instrumental conditioning might be both mediated by the same learning process(es) and subject to the same laws, such that variables known to affect the outcome of a classical contingency (by affecting the acquisition of signaling properties by the stimulus) may have a similar effect on the outcome of an instrumental contingency (by affecting the signal value acquired by the response). The reverse should also be true. According to such a signal theory, manipulations that affect the outcome of classical conditioning should similarly affect the outcome of instrumental conditioning (and vice versa), and phenomena within the domain of classical conditioning should be demonstrable as products of instrumental conditioning (and vice versa).

The pilot data for this research was supported by Grant APA 259 from National Research Council of Canada to N. J. Mackintosh, while the experiment here reported was supported by a 1975 grant from Wilfrid Laurier University. Requests for reprints should be sent to Robert St. Claire-Smith (Department of Psychology), Wilfrid Laurier University, Waterloo, Ontario, Canada.
In classical conditioning, the degree to which a stimulus becomes an effective CS depends importantly on the validity or reliability of that stimulus as a predictor of the UCS relative to that of other stimuli in the situation (Wagner, 1969). This conclusion derives mainly from studies of compound conditioning where it has been found that the acquisition of signal value by one of two stimuli reinforced in compound $\left(S_{1}\right)$ is reduced, or blocked (e.g., Kamin, 1968; 1969), to the extent prior training of the other stimulus alone $\left(S_{2}\right)$ has preestablished it as a valid signal of the UCS. A similar depreciation in $S_{1}$ signal value, sometimes termed overshadowing (e.g., Mackintosh, 1975), is found when either reinforced trials of $S_{2}$ alone (Dweck \& Wagner, reported by Wagner, 1969) or nonreinforced trials of $S_{1}$ alone (Egger \& Miller, 1962; Wagner, 1969) are interspersed with reinforced trials of the compound. In these latter instances, it is the preferential correlation of $S_{2}$ with the reinforcer, rather than a previous correlation, that establishes it as a more reliable predictor of the UCS than $S_{1}$.

A theory such as the one outlined above would suggest that the validity or reliability of the response as a predictor of a consequence might be similarly important to the outcome of an instrumental contingency. In a study reported elsewhere (St. Claire-Smith, 1979), a test of this prediction was conducted by attempting to demonstrate the overshadowing and blocking of punishment. Rats were trained to a stable rate of leverpress responding on a VI food-reinforce- 
ment schedule, given off-the-baseline classical conditioning sessions in which a brief stimulus (a light or a tone) was established as a CS for shock, and then subjected to a single session in which responses were occasionally punished by the shock. In the blocking condition, the established CS was presented coincident with each response followed by shock. Thus, each shock was preceded by a stimulus-response compound in which the stimulus was already established as a valid predictor of the shock. In the overshadowing condition, a neutral stimulus (the stimulus not experienced during classical conditioning) was presented coincident with each punished response. As most responses during the punishment sessions were not correlated with shock, this stimulus was the more reliable predictor of shock over the course of punishment training. Subjects in the control condition did not receive an external stimulus during punishment. The results were consistent with a signal theory interpretation. Responding was considerably suppressed by punishment in the control condition, much less so in the overshadowing condition, and still less in the blocking condition.

The present experiment was designed in an attempt to extend these findings by demonstrating the overshadowing of appetitive instrumental conditioning. Rats were trained to leverpress on a random-interval (RI) food-reinforcement schedule in which the reinforcer was delivered $450 \mathrm{msec}$ after a response. In the correlated condition, a $450-\mathrm{msec}$ stimulus (either a light or a tone) occurred in the interval between the response and the reinforcer. In one of the two control conditions, the uncorrelated condition, the brief response-produced stimulus was not correlated with reinforcement, while in the other control condition, there was no stimulus presented following responses. As the stimulus was preferentially correlated with food in the correlated condition, and therefore a more reliable signal of the reinforcer than responses, the rate of responding in this condition would be expected to be slower than in either of the control conditions. The stimulus should interfere with the acquisition of signal value by the response.

The experiment was also designed to permit an assessment of the extent to which the putative overshadowing stimulus became a signal for reinforcement over the course of RI training. A conditioned reinforcement test followed RI training during which the number of occurrences of a minimally trained alternate response (keypressing) over three extinction sessions was calculated. Subjects in the correlated and uncorrelated groups received the stimulus presented during RI training contingent on keypressing, while for subjects in the no-stimulus control group, such responses had no programmed effect. If the stimulus had become a signal for reinforcement in the correlated condition, it would be expected that these subjects would emit more keypress responses during the test than subjects in either of the other conditions (e.g., Egger \& Miller, 1962).

\section{METHOD}

\section{Subjects and Apparatus}

The subjects were 60 male hooded rats, of which 48 were used in the experiment, bred at the Charles River Laboratories, St. Constant, Quebec. They were about 120 days old on arrival and weighed between 250 and $280 \mathrm{~g}$. The animals were individually housed and, after 2 weeks of free access to food, were deprived to $75 \%$ of their ad-lib weight and maintained at that weight throughout the experiment.

The apparatus consisted of two single-lever rat chambers, $28 \mathrm{~cm}$ high, $30.5 \mathrm{~cm}$ long, and $25 \mathrm{~cm}$ deep, each enclosed in a soundattenuating shell. The floor consisted of .24-cm stainless steel rods, $1.27 \mathrm{~cm}$ center to center, the two side walls of clear Plexiglas, and the two end walls of aluminum. In addition to the lever located directly above the food magazine, each chamber contained a translucent white plastic key located to the right of the magazine $5 \mathrm{~cm}$ above the grid floor. The lever could be removed from the chamber or the key covered by a metal plate, as required. Ambient illumination was provided by a GE $7-\mathrm{W}$ frosted houselight situated behind the front panel. The brief light was produced by turning on a General Electric $40-\mathrm{W} \mathrm{Lu}$ miline bulb situated above the white Plexiglas ceiling to full intensity, while the 450-msec auditory stimulus was an 80 -dB tone (re $20 \mu \mathrm{N} / \mathrm{m}^{2}$ ) produced by a Mallery SC628 Sonalert tone generator. Reinforcement was the delivery of a $45-\mathrm{mg}$ Noyes pellet.

\section{Procedure}

The experiment was run in two replications, which differed only in the nature of the stimulus employed. In the first replication, the critical stimulus was the overhead light, while in the second, the 80-dB tone was used.

On the 1st day, keypress responding was established. The subjects were placed in the chambers, with the levers removed and 5 pellets in the magazine, and left until they had earned 30 pellets by pressing the key. (The subjects pressed the key with their noses.) Three subjects in the first replication and two in the second which failed to make the required 30 keypresses within $90 \mathrm{~min}$ were replaced. On the following day, leverpress responding was established, following the general procedure outlined above. The keys were covered and the subjects were left in the chambers until they had earned 100 pellets by pressing the lever. During each of the next 10 daily 30 -min sessions, leverpressing was reinforced on a random interval (RI) schedule with an expected mean interval value of $30 \mathrm{sec}$ (RI $30 \mathrm{sec}$ ). The probability of the first response in each successive 3-sec interval being reinforced was 1/10. There was a delay of $450 \mathrm{msec}$ between the critical response and the delivery of reinforcement.

For RI training, the 24 subjects in each replication were randomly assigned to one of three conditions. In the correlated condition, a 450-msec stimulus occurred in the delay interval between the response and reinforcement. In the uncorrelated condition, the brief response-produced stimulus was delivered on a second RI 30-sec schedule which operated independently of the RI reinforcement program. Subjects in the no-stimulus condition received neither the light nor the tone during RI training.

Following the completion of RI training, the chambers were returned to the keypress mode and all subjects received three 30-min daily sessions in which the stimulus experienced during RI training was presented following each keypress (there was no programmed consequence of responding for subjects in the no-stimulus condition). Food was withheld during these sessions. 


\section{RESULTS}

The results of RI training for the light and the tone replications are shown separately in Figure 1. A split-plot analysis of variance, with conditions (correlated vs. uncorrelated vs. no stimulus) as the between-subject factor and sessions as the withinsubject factor, was calculated for each replication. The analysis for the light replication revealed a significant effect due to conditions $[\mathrm{F}(2,21)=11.24$, $\mathrm{p}<.01]$ and sessions $[\mathrm{F}(9,189)=8.38, \mathrm{p}<.01]$, and a significant Condition by Sessions interaction $[F(18,189)=5.50, p<.01]$. After application of the Geisser-Greenhouse correction (Kirk, 1968), the sessions effect and the Conditions by Sessions interaction were still significant (with $p<.01$ and $p<.05$, respectively). A simple main-effects analysis of the Conditions by Sessions interaction indicated that the groups did not differ significantly in rate of responding on the first, the second, or the third session $(p>.10)$, but did differ significantly on each of the remaining seven sessions $(p<.05)$. There was a significant change in response rates over sessions in both the no-stimulus $[\mathrm{F}(9,189)=8.2, \mathrm{p}<.01]$ and uncorrelated $[\mathrm{F}(9,189)=14.0, \mathrm{p}<.01]$ conditions, and in the correlated condition $[F(9,189)=2.2$, $\mathrm{p}<.05]$. Further analysis, using Tukey's HSD test, indicated that the mean overall rate of responding in the correlated condition was significantly lower than in either of the control conditions $(p<.01)$, which did not differ from each other $(p>.10)$. From these analyses and inspection of the first panel in Figure 1, it can be concluded that the between-group differences in rate of responding was due to the fact that subjects in the two control conditions increased their rate of responding over sessions significantly faster than subjects in the correlated condition.

The analysis of variance of the tone replication found significant main effects of conditions $[\mathrm{F}(2,21)$ $=16.3, \mathrm{p}<.01]$, and sessions $[\mathrm{F}(9,189)=14.4$, $\mathrm{p}<.01]$, and a significant Conditions by Sessions interaction $[F(18,189)=6.2, p<.01]$. The sessions effect and the Conditions by Sessions interaction remained significant $(p<.01)$ after application of the Geisser-Greenhouse correction. The simple maineffects analysis of the interaction showed that the groups did not differ on the first session, but differed significantly in rate of responding from the second session on $(p<.05)$. Response rate changed significantly over sessions for the no-stimulus $[F(9,189)$ $=3.21, \mathrm{p}<.05]$, and uncorrelated $[\mathrm{F}(9,189)=9.9$, $\mathrm{p}<.01]$ groups, but not for the correlated group $[F(9,189)=1.4, p>.05]$. Tukey's HSD test indicated that the no-stimulus group and the uncorrelated group did not differ significantly in mean overall response rate $(p>.10)$, but that in both of these conditions subjects responded significantly faster than in the correlated condition $(\mathrm{p}<.01)$. It can be concluded from these analyses, in conjunction with inspection of the second panel in Figure 1, that in the tone replication, differences in rate of responding between the control groups and the correlated group emerged during RI training because response rates increased over sessions in the former groups but not significantly in the latter group.

During the course of RI training, a separate count was kept of the number of responses made during stimulus presentations in both the correlated and uncorrelated conditions. The purpose was to insure that the difference in responding between the two groups (if a difference was obtained) did not simply reflect a tendency for the subjects to respond in the presence of the stimulus in the uncorrelated condition but not in the correlated condition, where it would be expected that the stimulus would function as a discriminative cue to approach the food magazine. As expected, given the actual differences in responding that obtained, such a possibility can be discounted. While no responses occurred during a stimulus presentation in the correlated condition in either replication, only one such response was recorded in the light replication and only two were recorded in the tone replication in the uncorrelated condition.

Table 1 shows the mean number of keypresses made by the correlated, uncorrelated, and no-stimulus groups in each replication during the conditioned reinforcement test. A one-way analysis of variance revealed that between-group differences in total keypresses were significant for both the light $[\mathrm{F}(2,21)=$ $4.86, \mathrm{p}<.05]$ and the tone $[\mathrm{F}(2,21)=5.21, \mathrm{p}<.05]$ replications. Tukey's HSD test showed that for both replications there were no significant differences between the uncorrelated and no-stimulus control

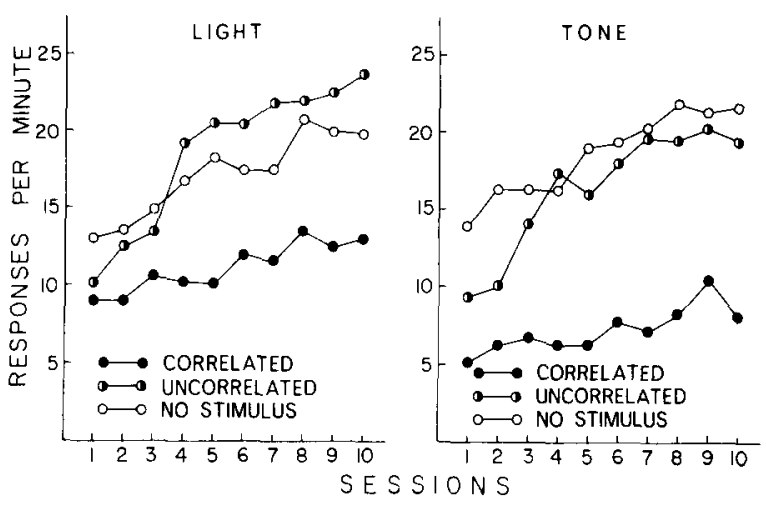

Figure 1. Mean response rates over the 10 RI 30 -sec training sessions for each of the three groups in the two replications. The correlated group received a stimulus during the $450-\mathrm{msec}$ delay between a response and reinforcement, the uncorrelated group recelved a 450 -msec stimulus following a response seldom correlated with reinforcement, while a stimulus never followed responding for the no-stimulus group. The stimulus was a light in the first replication and $a$ tone in the second. 
Table 1

Mean Number of Keypress Responses for the Three Groups in the Light Replication and the Tone Replication During the Conditioned Reinforcement Test

\begin{tabular}{lcc}
\hline & \multicolumn{2}{c}{ Replication } \\
\cline { 2 - 3 } Group & Light & Tone \\
\hline Correlated & 322 & 314 \\
Uncorrelated & 155 & 128 \\
No Stimulus & 138 & 146 \\
\hline
\end{tabular}

groups in mean number of keypresses made overall $(p>.10)$, but that the correlated subjects made significantly more keypress responses in each replication than did subjects in each of the control groups $(p<.05)$

\section{DISCUSSION}

The results of the present study show that the normal increase in rate of responding that occurs over successive RI training sessions is retarded when each occurrence of the response-contingent reinforcer is preceded by a stimulus better correlated with reinforcement than the response. An interpretation of this effect as an instance of overshadowing requires, of course, that the stimulus become a signal for the reinforcer during RI training. The data from the conditioned reinforcement tests demonstrate that this requirement was met: when contingent on keypressing presentation of the stimulus significantly increased the number of such responses emitted during extinction. This latter finding suggests one reason why leverpress responding in the correlated condition tended to increase rather than decline over RI sessions, although the possibility that overshadowing was simply incomplete in the present study cannot be discounted.

The findings of the present experiment complement those of the study described earlier (St. Claire-Smith, 1979), where it was found that the rate at which responding was suppressed as a function of FI punishment trials was slowed when each delivery of the response-contingent punisher was preceded by a stimulus, and are consistent with the results of several other experiments. Pearce and Hall (1978), in a set of experiments almost identical in design to the design of the present study, obtained essentially the same results as reported here. Rats increased their rate of leverpressing over variable-interval training sessions more rapidly when no stimulus occurred during a .5-sec delay between a response and food than when a light or a tone filled the delay interval. Williams (1975) reported that pigeons trained to peck a green key for food failed subsequently to learn to peck a red key for food if the food was delayed and the green key was illuminated during the delay inter- val. Subjects continued to respond on the green key, however, even though such responses now had no effect on the delivery of food. Williams interpreted his results as reflecting the blocking of the red key response-reinforcer correlation by the previously established green key response-reinforcer correlation. Thus, in both appetitive and aversive conditioning situations, the effectiveness of an instrumental contingency is reduced when another event in the environment is a more valid or reliable predictor of the outcome than the response. Further, the generality of this conclusion is not restricted to situations where the occurrence of the response is under the control of the subject or to situations where the response and competing signal occur sequentially. Konorski (1948, pp. 218-219) reported that a dog would cease raising its leg spontaneously following trials where the leg was lifted by the experimenter during the last $5 \mathrm{sec}$ of a 15 -sec stimulus and the dog was then fed, although forced leg raises followed by food in the absence of a competing signal resulted in an increase in voluntary leg raises during the intertrial interval. In the first instance, because spontaneous occurrences of the response were never paired with food, the stimulus was a more valid predictor of the reinforcer than the response.

Clearly the results of these experiments, and those of the present study, support interpretations of instrumental conditioning which emphasize learning about the consequences of responding (e.g., Bolles, 1972; Mackintosh, 1974) in addition to the signal theory advanced here. The emphasis within the latter position, however, is on the assumption of equivalence of classical and instrumental conditioning in terms of both learning processes and predicting events (i.e., the equivalence of responses and stimuli). It is the latter view that predicts the blocking and overshadowing of the acquisition of signal value by a stimulus if the US is more reliably predicted by a response.

\section{REFERENCES}

Bolles, R. C. Reinforcement, expectancy and learning. Psychological Review, 1972, 79, 394-409.

EgGen, M. D., \& Mrller, N. E. Secondary reinforcement in rats as a function of information value and reliability of the stimulus. Journal of Experimental Psychology, 1962, 64, 97-104.

Kamin, L. J. "Attention-like" processes in classical conditioning. In M. R. Jones (Ed.), Miami symposium on the prediction of behavior, 1967: Aversive stimulation. Coral Gables, Fla: University of Miami Press, 1968.

Kamin, L. J. Predictability, surprise, attention and conditioning. In B. A. Campbell \& R. M. Church (Eds.), Punishment and aversive behavior. New York: Appleton-Century-Crofts, 1969.

KIRK, R. E. Experimental design: Procedures for the behavioral sciences. Belmont, Calif: Wadsworth, 1968.

Konorski, J. Conditioned reflexes and neuron organization. Cambridge: Cambridge University Press, 1948. 
Mackintosh, N. J. The psychology of animal learning. New York: Academic Press, 1974.

Mackintosh, N. J. A theory of attention: Variations in the associability of stimuli with reinforcement. Psychological Review, 1975, 82, 276-298.

Pearce, J., \& Hall, G. Overshadowing the instrumental conditioning of a lever press response by a more valid predictor of reinforcement. Journal of Experimental Psychology: Animal Behavior Processes, 1978, 4, 356-357.

St. Claire-Smith, R. The overshadowing and blocking of punishment. Quarterly Journal of Experimental Psychology, 1979, 4, 51-61.
WaGner, A. R. Stimulus validity and stimulus selection in associative learning. In N. J. Mackintosh \& W. K. Honig (Eds.), Fundamental issues in associative learning. Halifax: Dalhousie University Press, 1969.

Williams, B. A. The blocking of reinforcement control. Journal of the Experimental Analysis of Behavior, 1975, 24, 215-225.

(Received for publication May 9, 1978; revision accepted October $5,1978$. 